\title{
ERRATUM
}

\section{Manipulation of the nuclear spin ensemble in a quantum dot with chirped magnetic resonance pulses}

Mathieu Munsch, Gunter Wüst, Andreas V. Kuhlmann, Fei Xue, Arne Ludwig, Dirk Reuter, Andreas D. Wieck, Martino Poggio and Richard J. Warburton

Nature Nanotechnology 9, 671-675 (2014); published online 24 August 2014; corrected after print 2 September 2014.

In the version of this Letter originally published, the first name of Gunter Wüst was misspelt. This error has now been corrected in the online versions of the Letter. 